\title{
Nonlinear Projective Filtering of ECG Signals
}

\author{
Marian Kotas \\ Silesian University of Technology, Institute of Electronics \\ Poland
}

\section{Introduction}

Since the beginnings of the noninvasive electrocardiology, development of the methods for reconstruction of the electrocardiogram (ECG) embedded in noise has stimulated the progress in the field. The predominant types of noise are baseline wander, powerline interference, electromyographic (EMG) noise and motion artifacts. Among them EMG and motion artifacts are the most difficult to be suppressed. It stems from the fact that their frequency spectra overlap that of the desired ECG, which makes the classical band-pass filtering ineffective. To mitigate the problem, the synchronized averaging was introduced. The method assumes that the ECG signal is repeatable and that the noise is additive, independent and of zero mean. Linear time-alignment (Jane et al. 1991) of the respective ECG beats and construction of an average one results in significant suppression of the noise; however, the inter-beat variability of the cardiac cycles is lost.

In many applications it is advantageous to suppress noise while preserving the variability of the desired signal morphology. In such cases, it is more advantageous to create a space of possible shapes instead of constructing an average template. This task can be accomplished with the help of principal component analysis (PCA) (Olmos et al., 1999; Kotas, 2006). In (Paul, 2000) the signal subspaces were constructed in the discrete cosine transform domain. However, modeling ECG beats with the help of the intrinsically linear PCA allows a limited success only (Kotas, 2006). In (Hu \& Nenov, 2006) construction of the signal subspaces was preceded with the nonlinear alignment of ECG beats. New capabilities emerged when the method of nonlinear state-space projections (NSSP) was applied to ECG processing (Schreiber \& Kaplan, 1996). Although this method also performs locally linear projections, globally the operation is nonlinear and allows more effective processing of the signal. This version of nonlinear projective filtering (NPF) was compared (Hu \& Nenov, 2006) to several other sophisticated methods of ECG noise suppression and achieved the best results in EMG noise environment. NPF allows not only a kind of smoothing of ECG signals but can also be used for separation of signal components with overlapping spectra. It was successfully applied (Richter et al., 1998) to fetal ECG extraction from the maternal abdominal signals.

Although NSSP method offers so advantageous performance, its practical applications are limited by the high computational costs. In (Kotas, 2004) linear time-alignment of the ECG beats was used to decrease the number of the constructed signal subspaces. In the conditions tested, projective filtering of time-aligned ECG beats (PFTAB) was several hundreds faster than NSSP. Moreover, PFTAB appeared rather effective in EMG noise 
environment. This method can be regarded as an extension of time averaging, preserving the deviations of the individual beats from the average one. However, when the length of the individual beats and the positions of the low amplitude ECG waves are highly variable, its performance can decrease. The method of dynamic time warping (Sakoe \& Chiba, 1978) helped to overcome the problem. Projective filtering of time-warped ECG beats (PFTWEB) appeared much more effective in such conditions (Kotas, 2008b).

Application of nonlinear projective filtering to ECG reconstruction prior to the analysis of the ventricular repolarization significantly improves the accuracy of the measurements (Kotas, 2007a). The ability of the method to extract the fetal ECG from the maternal abdominal electric signals was demonstrated in (Kotas, 2007b).

The aim of this study is to reveal the fundamental differences among the mentioned versions of the nonlinear projective filtering and to present how these differences influence the NPF performance. In sections 2, 3 and 4 the necessary details of the compared methods operation are reminded. The differences among the methods and their applications are studied in section 5 . The final conclusions are formulated in section 6 .

\section{Nonlinear state-space projections}

The method was proposed by (Schreiber \& Kaplan, 1996) as an effective tool for suppression of the wide-band electromyographic noise, contaminating the ECG signals. It forms the state-space representation of the observed noisy signal by application of the embedding operation (Schreiber \& Kaplan, 1996). A point in the embedding space is a vector:

$$
\mathbf{x}^{(n)}=\left[x(n), x(n+\tau), \ldots, x(n+(m-1) \tau]^{\mathrm{T}}\right.
$$

where $x(n)$ is the processed signal, $\tau$ is the time lag ( $\tau=1$ in this application), $m$ is the embedding dimension.

For each point $\mathbf{x}^{(n)}$ a small neighborhood $\Gamma^{(n)}$ is constructed, composed of the points which are close to $\mathbf{x}^{(n)}$

$$
\Gamma^{(n)}=\left\{k:\left\|\mathbf{x}^{(k)}-\mathbf{x}^{(n)}\right\| \leq \varepsilon\right\}
$$

where $\|$.$\| denotes the distance, \varepsilon$ is the radius of the neighborhood (Schreiber, 1995).

Within each neighborhood the local mean is computed (the neighborhood mass center)

$$
\overline{\mathbf{x}}^{(n)}=\frac{1}{\left|\Gamma^{(n)}\right|} \sum_{k \in \Gamma^{(n)}} \mathbf{x}^{(k)}
$$

$\left(\left|\Gamma^{(n)}\right|\right.$ denotes the cardinality of $\left.\Gamma^{(n)}\right)$ and the covariance matrix $\mathbf{C}^{(n)}$ of the neighborhood points deviations from the mass center (Schreiber \& Kaplan, 1996). Then the transformed version of the covariance matrix is calculated $\mathbf{G}(n)=\mathbf{R} \mathbf{C}^{(n)} \mathbf{R}$. $\mathbf{R}$ is a diagonal penalty matrix introduced to penalize the corrections of the first and the last coordinates of $\mathbf{x}^{(n)}\left(r_{1,1}=r_{\mathrm{m}, \mathrm{m}}=\right.$ $r$, where $r$ is large (Schreiber \& Kaplan, 1996), and the other diagonal entries of $\mathbf{R}$ are equal to 1 ). The transformed covariance matrix undergoes eigendecomposition, and the calculated 
eigenvectors $\mathbf{e}_{i}^{(n)}$ corresponding to the largest eigenvalues are used to compose the so-called signal subspace. After constructing the subspace of the assumed dimension $q(q<m)$ the point $\mathbf{x}^{(n)}$ is projected into this subspace

$$
\mathbf{x}^{(n)}=\overline{\mathbf{x}}^{(n)}+\mathbf{R}^{-1} \mathbf{E}_{q}^{(n)} \mathbf{E}_{q}^{(n) \mathrm{T}} \mathbf{R}\left(\mathbf{x}^{(n)}-\overline{\mathbf{x}}^{(n)}\right)
$$

where $\mathbf{E}_{q}(n)=\left[\mathbf{e}_{1}^{(n)}, \ldots, \mathbf{e}_{q}^{(n)}\right]$.

The projection is performed for each trajectory point. Since each sample of the processed signal occurs in $m$ points, its correction is a result of $m$ individual corrections. It can be calculated as their average (Schreiber \& Kaplan, 1996).

A
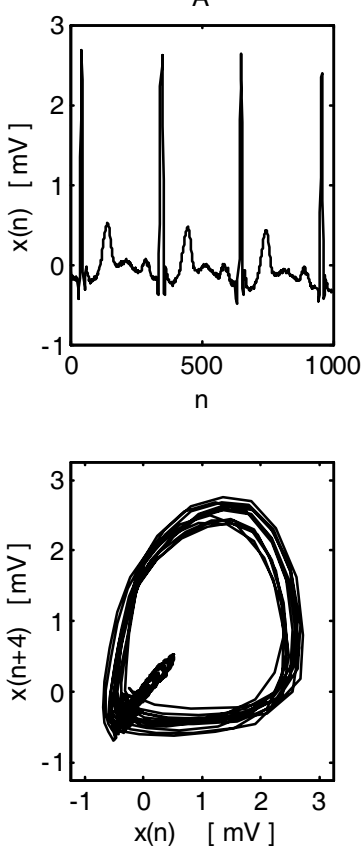

B
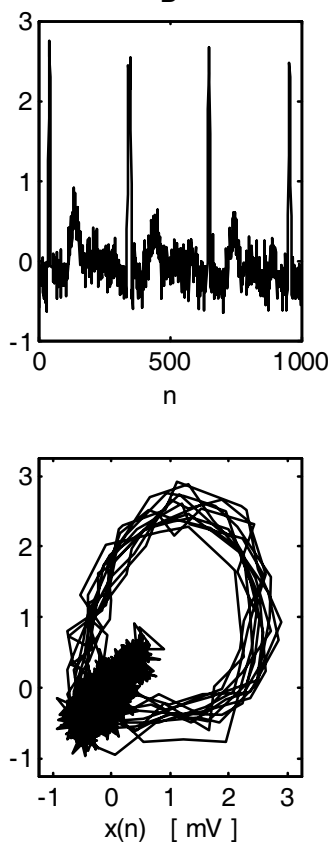

C
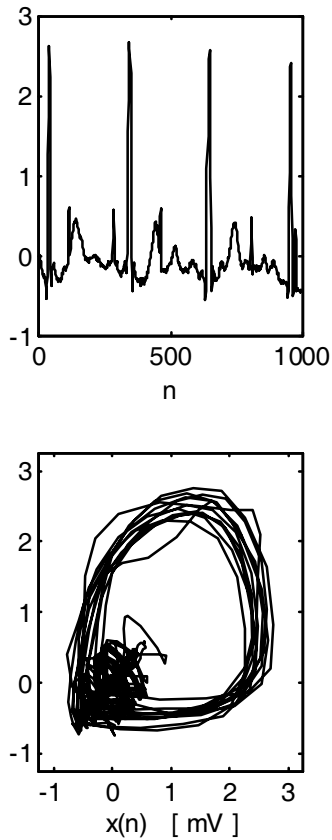

Fig. 1. Embedding space trajectories obtained for: a high quality ECG (A), the ECG with white Gaussian noise (B) and the simulated signal of a pregnant woman (C).

Since high dimensional embedding cannot be shown graphically, two-dimensional trajectories were presented in Fig.1. Although for noise reduction much higher embeddings are required, even such two-dimensional representation reveals some interesting properties of the ECG signal state-space structure. The trajectory of a high quality ECG (A) is close to a nonlinear manifold. For the same desired ECG contaminated with white Gaussian noise, the trajectory fluctuates around the "clean" one. Similarly, addition of the fetal ECG (C) results in the appearance of small loops circulating around the "clean" trajectory. 
The described method (NSSP) is aimed to create locally linear signal subspaces for the respective points of the state-space trajectory. By projecting the points into these subspaces we shift the trajectory towards the "clean" one and, as a result, we suppress noise preserving the fluctuations of the desired ECG. Averaging of locally linear projections results in globally nonlinear transformation of the trajectory. Similar transformation of the trajectories obtained for the maternal abdominal signals (see Fig.1 C) results in suppression of the fetal electrocardiogram (FECG) and estimation of the maternal ECG (MECG). To extract the FECG, we simply subtract the estimated MECG from the original abdominal composite signal.

The ability of NSSP to suppress the wide-band ECG noise while preserving the desired component morphological variability and even to separate the signal components of similar spectral properties (MECG and FECG) is a great advantage. Another advantage is that the method is very general. It can be applied without a complicated adjustment of its action to processing of different signals. However, its practical applications are limited by the extremely high computational costs (resulting from the fact that for each point of the statespace trajectory we have to determine the neighborhood and to create the signal subspace individually). To reduce these computational costs, the approach preceded by ECG beats time-alignment was developed (Kotas, 2004). This operation allows limiting the number of the constructed signal subspaces to the number of different positions within a beat, and the points occupying the same position within different beats can be projected into the same subspace.

\section{Projective filtering of time-aligned ECG beats}

In this method, construction of signal subspaces and state-space projections are preceded (Kotas, 2004) with the following operations:

- Linear filtering for baseline wander and power line interference suppression (Wariar \& Eswaran, 1991),

- QRS complex detection (Hamilton \& Tompkins, 1986),

- Beats classification as either dominant or aberrant (Pahlm \& Sörnmo 1987): the latter are excluded from further analysis, as well as the beats preceded or followed by aberrant ones,

- Cross-correlation function based synchronization of the detected complexes.

The last operation produces a set of fiducial marks $\left\{r_{k} \mid k=1,2, \ldots, K\right\}$ corresponding to the same position within the respective detected QRS complexes. Once the fiducial marks are established, the intrinsic operations of the method can be realized.

Each beat begins the assumed number of samples before its fiducial mark and ends one sample before the beginning of the next beat (Kotas, 2004). In order to facilitate construction of local signal subspaces (LSS), the respective beats are stored in an auxiliary matrix $\mathbf{T}$ (although in this method the term local refers to the position within a beat, it is also related with the points location in the embedding space). Each beat occupies one column of $\mathbf{T}=\left[\mathbf{t}_{k}\right]_{k=1}{ }^{k=K}$. The number of rows (which is denoted as $I$ ) depends on the length of the longest beat $\left(R R_{\max }\right)$. It must be large enough to allow construction of a signal subspace for the position $j=R R_{\max }$. Thus $I=R R_{\max }+(m-1)$, and all the beats in $\mathbf{T}$ are extended to this length - the method of zero order extension, which extends a beat by repeating its last sample (Chou, 2006), is applied. 
Time-alignment of the beats enables easy determination of LSS corresponding to the respective positions within a beat. To this end, for each $j\left(1 \leq j \leq R R_{\max }\right)$ a submatrix of $\mathbf{T}$ is selected (Kotas, 2004)

$$
\mathbf{T}^{(j)}=\left[\mathbf{t}_{k}{ }^{(j)}\right]_{k=1}{ }^{k=K}=\left[t_{i, k}{ }^{(j)}\right]_{i, k=1}{ }^{i=m, k=K} \text {, where } t_{i, k}^{(j)}=t_{i-1+j, k}
$$

containing the vectors $\mathbf{t}_{k}(j)$, which correspond to the synchronized trajectory points.

To make construction of signal subspaces more robust against outliers, we form a local neighborhood $\Gamma^{(j)}$ by rejecting the assumed fraction $c_{R}$ of the most distant points. After determination of the local neighborhood, the local mean $\overline{\mathbf{t}}(j)$ is computed and the covariance matrix $\mathbf{C}^{(j)}$ - of the deviations from the mean. In projective filtering of time-aligned ECG beats, the concept of penalizing the corrections of the first and the last coordinates of the embedding space vectors was desisted (Kotas, 2004). A local signal subspace corresponding to the $j$ th neighborhood is calculated by eigendecomposition of the covariance matrix $\mathbf{C}(j)$. After creating the signal subspaces for the respective positions within an ECG beat (the learning phase of PFTAB), the processing phase begins. It consists of the following steps: determination of a position $j$ within a beat, the point under correction occupies; projecting the considered point into the corresponding signal subspace; averaging of the results of the respective points projection (Kotas, 2004).

The introduced modifications significantly decreased the computational costs of NSSP. Moreover, PFTAB appeared not only much faster, but also rather effective in processing the ECG signals disturbed by EMG noise (the influence of noise on determination of neighborhoods was cancelled). This property appeared particularly advantageous when application of projective filtering to fetal ECG extraction was studied (Kotas, 2007b). Unfortunately, high fluctuations of the cycles length and large temporal shifts of the low amplitude ECG waves can decrease PFTAB performance. Therefore a new version of nonlinear projective filtering was developed (Kotas 2008b). In this version, the method of dynamic time warping (Sakoe \& Chiba, 1978) was applied to neighborhoods determination.

\section{Projective Filtering of Time Warped ECG Beats}

The preprocessing steps of the method are the same as in PFTAB. They produce a set of fiducial marks $\left\{r_{k} \mid k=1,2, \ldots, K\right\}$ corresponding to the same position within the respective detected QRS complexes. The embedding space points are divided into successive, slightly overlapping sequences of vectors whose time indices belong to the following sets

$$
\Psi_{k}=\left\{a_{k}+l \mid l=0,1, \ldots, N_{k}\right\}, \quad k=1,2, \ldots, K-1
$$

where

$$
a_{k}=r_{k}-b-\frac{m}{2}, \quad N_{k}=r_{k+1}-r_{k}+2 b
$$

$b \in N$ is a small number introduced to immunize the method against small errors of the fiducial marks determination (Kotas, 2008b). 


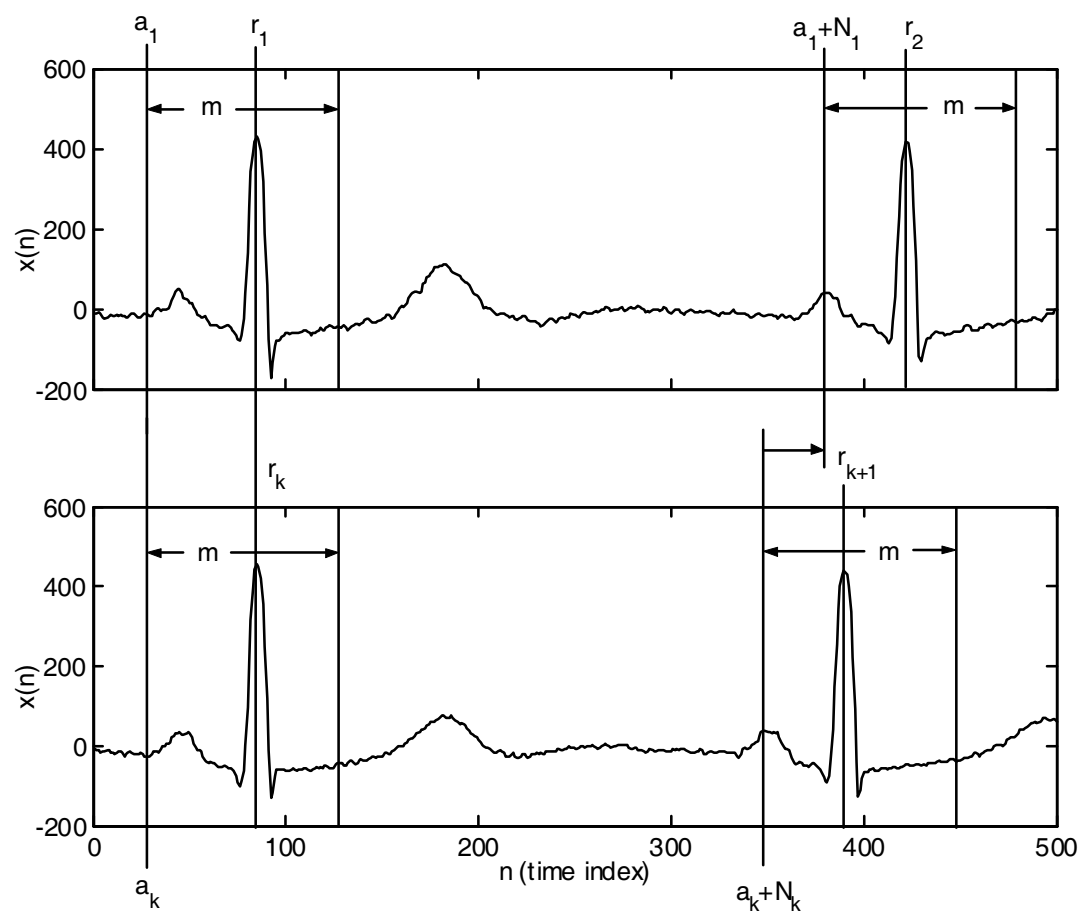

Fig. 2. The ECG signal sections containing successive QRS complexes. The presented delay windows show the first and the last state-space points of $\Psi_{1}$ and $\Psi_{\mathrm{k}}$ respectively. The $k \mathrm{th}$ sequence of points is time warped with respect to the first one.

The general concept (Kotas, 2008b) is to perform the nonlinear alignment of the successive sequences of vectors $\left(\Psi_{k}, k=2,3, \ldots K-1\right)$ with respect to the first sequence and this way to determine the neighborhoods corresponding to the respective positions within $\Psi_{1}$.

For small $b$ the side points $\mathbf{x}^{\left(a_{1}\right)}$ and $\mathbf{x}^{\left(a_{1}+N_{1}\right)}$ overlap QRS complexes (see Fig.2), and thus their neighborhoods can be determined on the basis of the preprocessing step results:

$$
\begin{aligned}
& \Gamma^{\left(a_{1}\right)}=\left\{a_{k} \mid k=1,2, \ldots, K-1\right\} \\
& \Gamma^{\left(a_{1}+N_{1}\right)}=\left\{a_{k}+N_{k} \mid k=1,2, \ldots, K-1\right\}
\end{aligned}
$$

Determination of the neighborhoods corresponding to the respective positions within $\Psi_{1}$ is performed in the following way (Kotas, 2008b).

1) In the first step each neighborhood of a point from $\Psi_{1}$ is filled with this point only, and so the time index of this point is included in the neighborhood set

$$
\Gamma^{\left(a_{1}+l\right)}=\left\{a_{1}+l\right\}, \quad l=0,1, \ldots, N_{1}
$$


These points become the first mass centers of the respective neighborhoods. Then we set $k=2$ and go to the next step.

2) The sequences of points that belong to $\Psi_{k}$ are time warped with respect to the sequence of the mass centers. For this purpose, the so-called warping paths are calculated: $w_{k, l}, l=0,1, \ldots, N_{1}$, containing the indices of the vectors $\mathbf{x}^{\left(a_{k}+w_{k, l}\right)}$ that are aligned with the successive mass centers $\overline{\mathbf{x}}^{\left(a_{1}+l\right)}$. The warping path is calculated by minimizing

$$
Q_{k}=\sum_{l=0}^{N_{1}} \| \mathbf{x}^{\left(a_{k}+w_{k, l}\right)}-\overline{\mathbf{x}}^{\left(a_{1}+l\right) \|}
$$

while preserving the border conditions, the monotonicity conditions and the condition restricting the number of successive points from $\Psi_{k}$ that may be omitted in the warping path (Kotas, 2008a). The minimization is performed with the use of the dynamic programming (Sakoe \& Chiba, 1978).

3) After the $k$ th sequence $\Psi_{k}$ has been time warped with respect to the sequence of the mass centers, the neighborhoods are supplemented with the points whose indices are stored in the warping path

$$
\Gamma^{\left(a_{1}+l\right)}=\Gamma^{\left(a_{1}+l\right)} \cup\left\{a_{k}+w_{k, l}\right\}
$$

4) The mass centers of the respective neighborhoods are updated and $k$ is incremented. If $k<K-1$ we go to step 2, otherwise we end the algorithm.

After determination of the respective neighborhoods, the corresponding signal subspaces are constructed. As in PFTAB, to make the constructed subspaces robust against outliers, in each neighborhood the assumed fraction $c_{R}$ of the most distant points is rejected. Construction of signal subspaces for the respective positions within $\Psi_{1}$ ends the learning phase of PFTWEB. The processing phase, as in PFTAB, consists of state-space points projecting and introduced corrections averaging - for details see (Kotas, 2008b).

\section{Results}

In this section, we will first observe and analyze the differences among the compared versions of nonlinear projective filtering. Then we will study the chosen versions ability to suppress noise or to separate the signal components with overlapping spectra.

\subsection{Distributions of neighborhoods points}

Different approaches to neighborhoods determination result in different contents and properties of the constructed neighborhoods. 


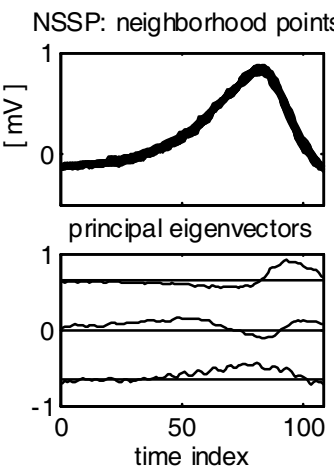

3D principal subspace

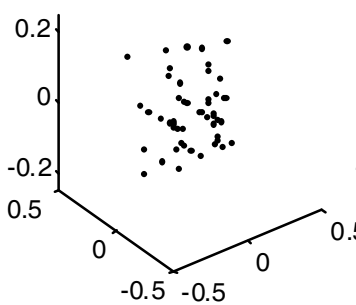

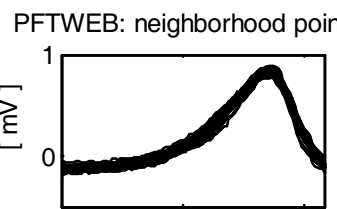

principal eigenvectors

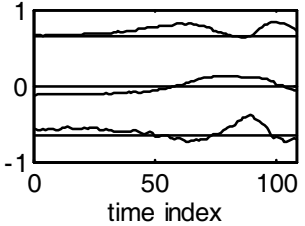

3D principal subspace

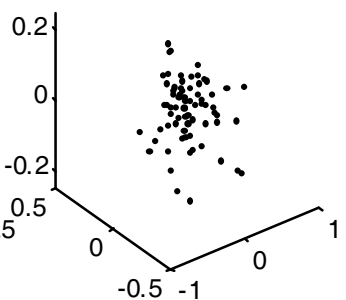

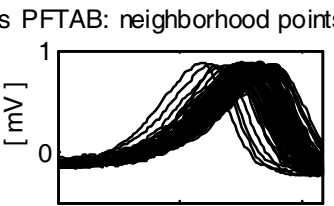

principal eigenvectors

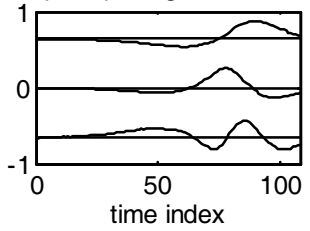

3D principal subspace

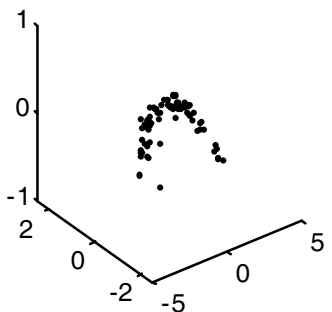

Fig. 3. The neighborhoods determined by the respective methods while processing a high quality signal with a shifting $\mathrm{T}$ wave. The uppermost are one-dimensional representations of the neighborhood points; below, the first three principal eigenvectors - vertically shifted; below, distributions of the neighborhoods points in 3D principal subspaces.

In Fig. 3 we can see that one-dimensional representations of the points included into the neighborhood by NSSP are very close to each other. Their dispersion in the threedimensional principal subspace is very low. The neighborhood was determined to perform reconstruction of one state-space point only. Since this point must be located close to the center of the presented cloud of points, a high precision of reconstruction is possible even for a very low dimension of the signal subspace.

The neighborhood determined by PFTAB is of much higher dispersion. It is caused by different positions of the individual $\mathrm{T}$ waves within the ECG beats. The characteristic structure of the points three-dimensional representation reveals nonlinear relations among them. For so low level of noise, higher variability of the desired component assured determination of very smooth principal eigenvectors. However, since many points of the neighborhood are located very far from the mass center, much higher dimension of the signal subspace is necessary for their precise reconstruction.

Applying nonlinear alignment of the sequences of state-space vectors (PFTWEB), we achieved relatively precise synchronization of the $\mathrm{T}$ waves. Although the dispersion of points is larger than that achieved by NSSP, it is much smaller than that obtained by PFTAB. As a result, the dimension of the signal subspace doesn't have to be so high as for PFTAB. 
NSSP: neighborhood points PFTWEB: neighborhood points PFTAB: neighborhood points
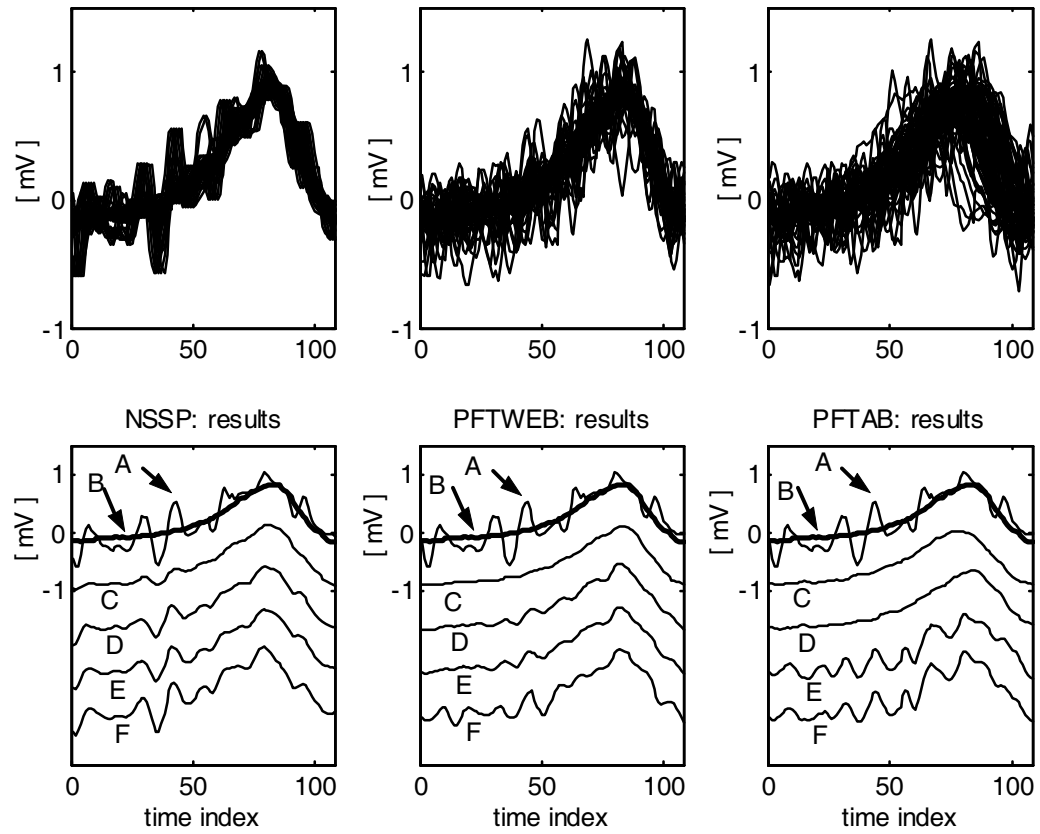

Fig. 4. Results of a state-space point reconstruction in the simulated EMG noise environment: $\mathrm{A}$ - the point under reconstruction; $\mathrm{B}$ - the desired component of $\mathrm{A}$; $\mathrm{C}$ - the neighborhood mass center; $\mathrm{D}, \mathrm{E}, \mathrm{F}$ - results of reconstruction for the signal subspace dimension of 1, 2 and 3 respectively (C,D,E,F are vertically shifted).

The influence of real electromyographic, highly correlated noise on state-space points reconstruction was illustrated in Fig. 4. For NSSP not only the desired, but also the noise component had a great impact on neighborhood determination (the noise deviations from the desired component are well synchronized). As a result, even the neighborhood mass center (C) contains a discernible noise component. With increasing dimension of the signal subspace, suppression of noise was less and less effective.

For PFTAB the noise components of the neighborhood points are not synchronized and calculation of the mass center caused their effective suppression. Although the mass center (C) is shifted with respect to the desired component (B), for $q=1$ relatively precise reconstruction was achieved - with effective suppression of noise.

Application of dynamic time warping allowed more flexible determination of the neighborhood with rather low influence of noise. It improved calculation of the neighborhood mass center, which is visually free of noise and closer to the desired component (B) than the mass center constructed by PFTAB. However, for increasing $q$ the suppression of noise was less and less effective.

Separation of the maternal and the fetal ECG is even more difficult than suppression of EMG noise. In the first stage, projective filtering is applied to suppress the fetal component 
and to estimate the maternal ECG. In the second stage, the estimated MECG is subtracted from the original composite signal and this way suppressed. As a result, the fetal component is extracted. However, when the estimated MECG contains some residua of the fetal QRS complexes, its subtraction causes inconvenient suppression of the fetal ECG.
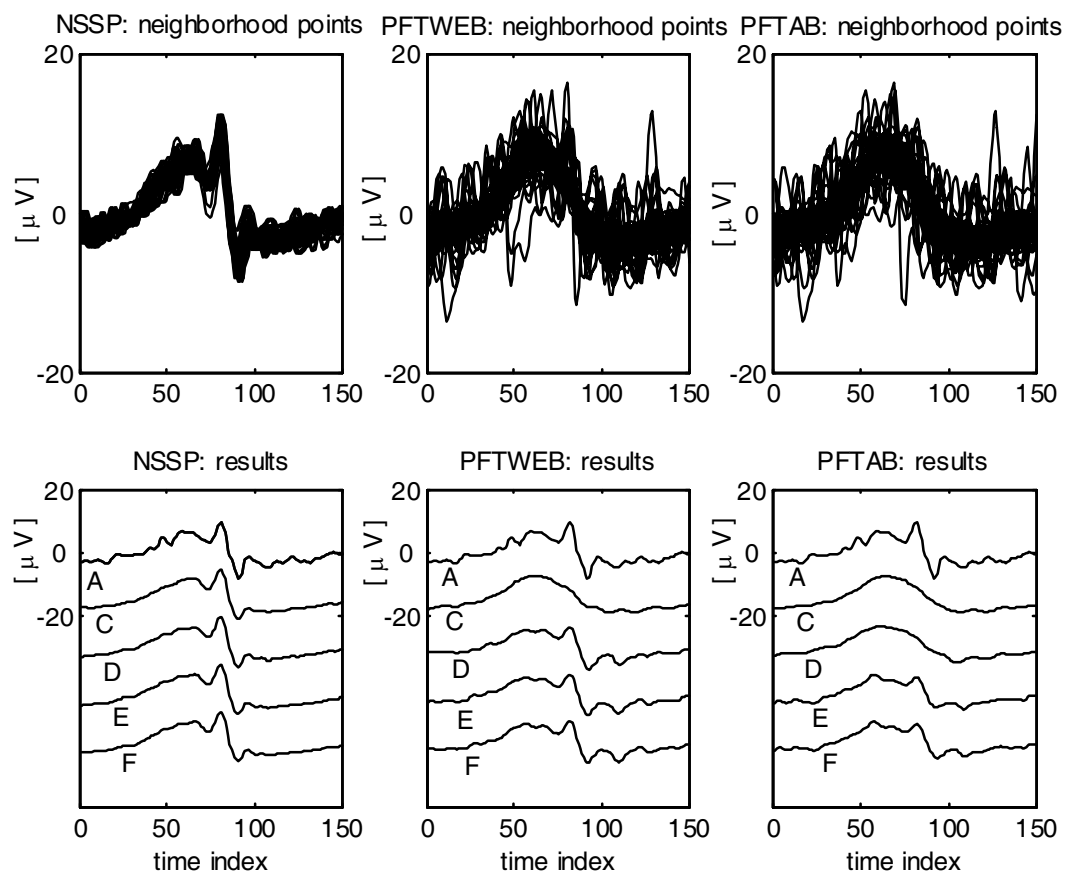

Fig. 5. Results of a state-space point reconstruction for the real maternal abdominal signal (the point corresponds to the maternal T wave with the overlapping fetal QRS complex). The further description is as in Fig.4. For this signal the desired component (B) was not available.

Reconstruction of the point corresponding to the maternal $\mathrm{T}$ wave with the overlapping fetal QRS complex is illustrated in Fig. 5. In this case NSSP failed. Most points of the neighborhood contained a fetal QRS complex and were synchronized with respect to it. As a result, the complex was preserved in the neighborhood mass center (C) and, consequently, the state-space projection could not have caused its suppression. For PFTWEB the complex was suppressed by calculation of the mass center. However, projections into the principal subspaces of different non-zero dimension resulted in its reconstruction. Only PFTAB allowed suppressing the fetal complex for non-zero dimension of the signal subspace.

Although many factors influence state-space points reconstruction, and in different conditions the results can be quite different, the presented cases were chosen to illustrate the most typical action of the compared methods. We can conclude that PFTWEB is most appropriate for ECG enhancement in EMG noise environment, but PFTAB allows most effective extraction of the fetal ECG. 

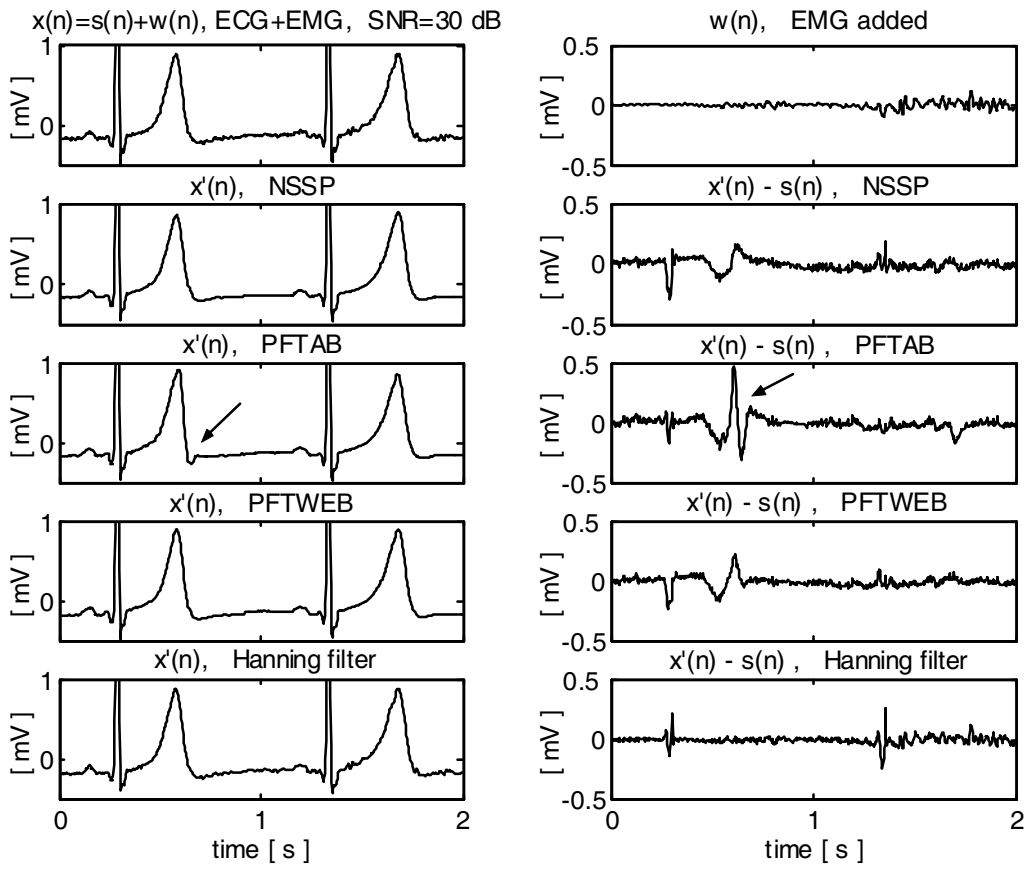

Fig. 6. Results of ECG signal filtering in a case of the significantly shifted $\mathrm{T}$ wave. The arrows indicate poor results obtained by PFTAB.

\subsection{Suppression of noise versus precision of the desired ECG reconstruction}

The most important parameters of the projective filters are: embedding dimension $(m)$ and the dimension of signal subspaces $(q)$. When the level of noise is low, it is crucial to assure high precision of the desired component reconstruction. To achieve it, we can either increase $q$ or decrease $m$. For higher level of noise, however, it is very important to assure its effective suppression. It can be achieved by decreasing $q$ or increasing $m$. In (Kotas, 2008c) the parameters balancing both requirements (for $\mathrm{SNR}=10 \mathrm{~dB}$ ) were chosen: $\tau_{m}=500 \mathrm{~ms}$ and $q=1$ for NSSP; $\tau_{m}=200 \mathrm{~ms}$ and $q=2$ for PFTAB; $\tau_{m}=400 \mathrm{~ms}$ and $q=2$ for PFTWEB $\left(\tau_{m}\right.$ is the embedding window width, corresponding to $m$, independent from the sampling frequency applied).

The precision of the desired component reconstruction, offered by the respective filters for the above parameters, is illustrated in Fig.6. For reference the results obtained by application of the low-pass Hanning filter $\left(\mathrm{H}(\mathrm{z})=\left(\mathrm{z}+2+\mathrm{z}^{-1}\right) / 4\right)$ are presented. The processed signal characteristic feature is high variability of the QT interval. Particularly the first T wave in the presented signal segment is considerably shifted to the left. For $q=2$, PFTAB was not able to reconstruct the shifted wave precisely. Much better results were achieved by both NSSP and PFTWEB.

Analyzing the residual noise $\left(x^{\prime}(n)-s(n)\right)$ obtained for NSSP and PFTWEB, we can notice that the first QRS complex was reconstructed with lower precision than the second one. It 
was caused by the influence of the shifted $\mathrm{T}$ wave of high amplitude on determination of neighborhoods for the points overlapping both the QRS complex and the T wave. For the first complex in the figure, the amplitude of the residual noise is comparable for all filters applied. However, for the second one, the Hanning filter introduces much higher disturbances than any of the three projective filters compared.

$x(n)=s(n)+w(n), E C G+E M G, S N R=10 d B$
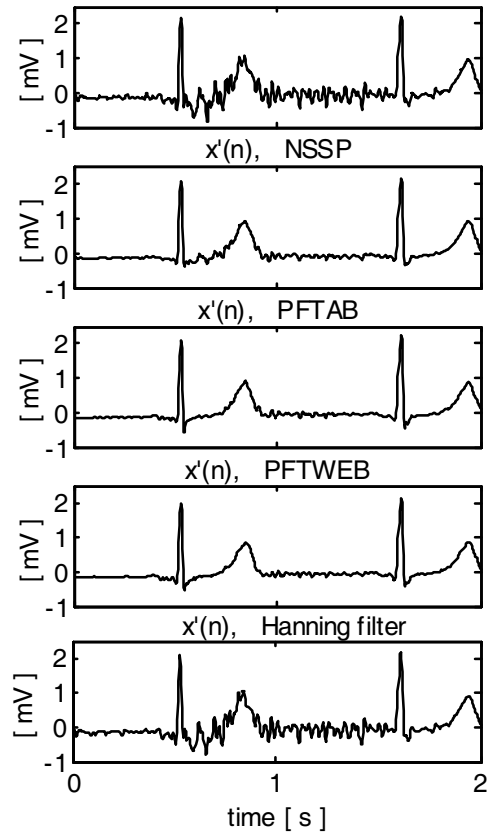
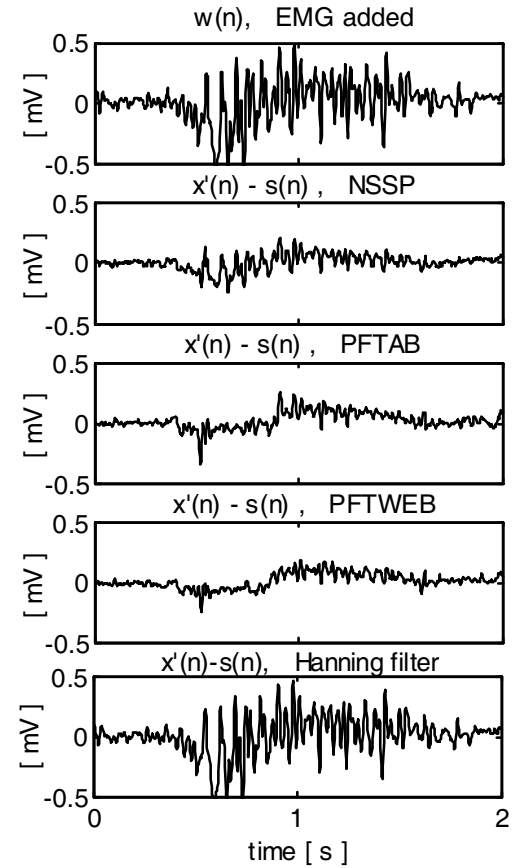

Fig. 7. Results of ECG signal filtering for a moderate level of the EMG noise.

The ability of the projective filters to suppress the EMG noise was illustrated in Fig.7. In the presented example, PFTWEB performed best, but PFTAB achieved rather comparable results. NSSP caused significant suppression of noise as well; however, for this filter energy of the residual EMG noise was clearly higher. The Hanning filter, which introduces similar distortions of the QRS complex as the projective filters (see Fig.6), caused hardly any suppression of the EMG noise. Concluding, nonlinear projective filtering allows much more effective processing of the ECG signals, with higher precision of the desired component reconstruction and better suppression of noise. Among the compared versions of NPF, the best compromise between both requirements was achieved by PFTWEB.

\subsection{NPF for reliable evaluation of the repolarization duration (RD) variability}

The time between depolarization and repolarization of the ventricles is covered by the QT interval (from the QRS onset to the T wave end). It is an important electrocardiographic parameter, often used to quantify the duration of ventricular repolarization (Malik, 2000). 
Since precise determination of the QRS onset and the T wave end is relatively difficult, new variables were defined to evaluate the repolarization duration variability. The problem is discussed at length in (Merri et al., 1993; Tikkanen et al., 1999). According to (Tikkanen et al., 1999) the most precise measurement of $\mathrm{RD}$ can be obtained if the left limit of the repolarization interval is defined as the position of the $R$ wave maximum $(R)$ and the right limit as the position of the T wave peak (Tp). According to (Merri et al., 1993) the RTp interval is highly correlated with the actual QT interval; therefore, it can be used for the analysis of repolarization duration variability. In (Kotas 2008c) the experiments with the latest version of the nonlinear projective filtering were performed. NPF improved evaluation of RD variability both in time and frequency domain. This area of projective filtering applications is discussed in this subsection.
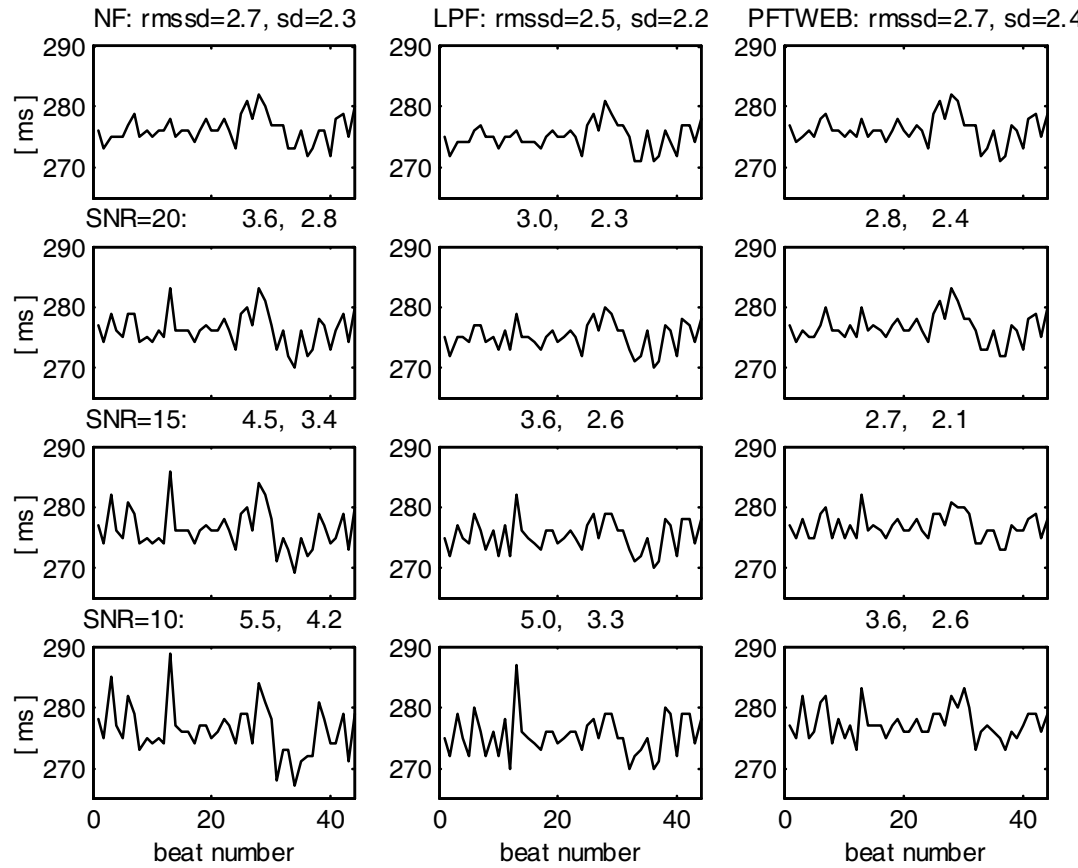

Fig. 8. The RTp series determined on the basis of a high quality ECG (the uppermost) and the ECG with EMG noise added (below). Each series is evaluated with two indices of variability: SD and RMSSD - defined by (12). The columns correspond to the filtering operations executed before the measurements (NF denotes "not filtered").

Fig.8 presents the RTp series determined on the basis of a high quality ECG from the QT database (Laguna et al., 1997) and for the signals with EMG noise added. For the localization of the T wave peak, the procedure developed by (Laguna et al., 1990) and modified by (Tikkanen et al., 1999) was used; for the precise description - see (Kotas, 2007a). The procedure was applied to the original (not filtered) signals and to the signals enhanced by different operations. Since the measurements of the repolarization duration can be 
performed for high quality signals only, and the precision of the desired signal reconstruction is of the primary interest, PFTWEB was applied with $q=3$ for $\tau_{m}=400 \mathrm{~ms}$ (the dimension of the signal subspaces is higher than in the previous experiments). For reference the low-pass filter with the cut-off frequency of $15 \mathrm{~Hz}$, which had appeared effective in (Kotas 2008c), was employed.

A) RTp series (no EMG added, NF)
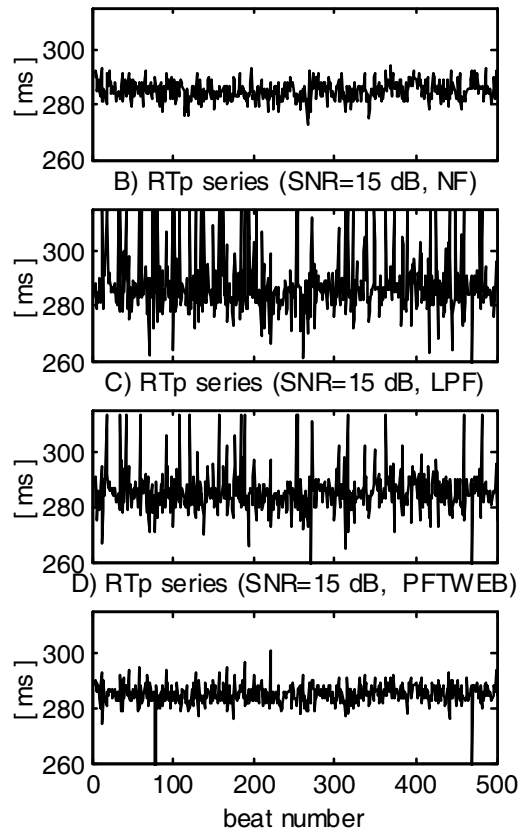

A) PSD ${ }_{\text {RT }}$

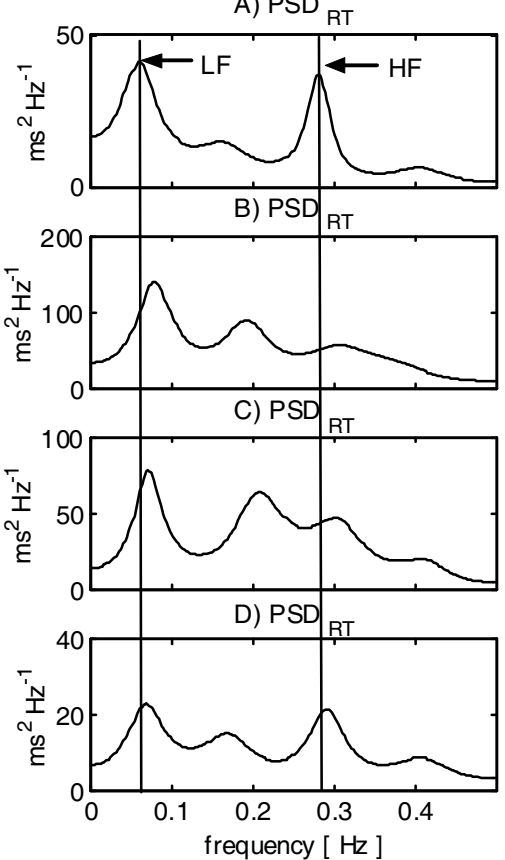

Fig. 9. Spectral analysis of RTp interval variability: A) the series obtained for a high quality ECG; B,C,D) the series obtained after different filtering operations, for the signal with EMG added. LF and HF are the low and the high frequency peaks of the true PSD (of series A).

The determined time series are evaluated with two indices of variability: the standard deviation (SD) and the root mean square of the successive differences (Tikkanen et al., 1999)

$$
\operatorname{RMSSD}_{x}=\sqrt{\frac{1}{N-1} \sum_{n=1}^{N-1}(x(n+1)-x(n))^{2}}
$$

where $x(n), n=1,2, \ldots, N$ is the analyzed time series.

We can notice that addition of even very weak noise $(S N R=20 \mathrm{~dB})$ raises the errors of the measurements performed on the original not filtered (NF) signals. Since the RD measurement errors increase the RD series variability, for the growing level of noise the variability indices grow as well. Application of low-pass filtering slightly decreased the 
measurement errors and the growth of the indices. However, PFTWEB allowed much more precise measurements and, as a result, most reliable evaluation of the RTp series variability in time domain.

The PFTWEB influence on spectral analysis of the RTp intervals variability was illustrated in Fig.9. The uppermost on the left is the series established on the basis of a high quality ECG signal from the QT database. When the signal was contaminated by the EMG noise of highly variable level (the average $S N R=15 \mathrm{~dB}$ ), localization of the $\mathrm{T}$ wave maximum failed in numerous cases. As a result, series B is rather jagged - corrupted by large measurement errors. Preceding the measurements with low-pass filtering slightly improved the measurements - the number of large errors decreased. However, application of PFTWEB caused much more significant improvement of the results. Only a few large errors of RTp interval determination occurred.

For each RTp series determined, the following operations were performed: large measurement errors were detected and replaced by the preceding values, the corrected series was resampled with the frequency of $1 \mathrm{~Hz}$ (by application of first order interpolation) and then filtered to remove the low frequency trend (the Butterworth high-pass filter with the cut-off frequency of $0.02 \mathrm{~Hz}$ was applied in forward and reverse direction). The power spectra were estimated by application of the autoregressive modeling (Lim \& Oppenheim, 1988) with the model order equal to 10.

The repolarization interval series often contain the oscillatory components of low frequency (LF) - close to $0.1 \mathrm{~Hz}$ - related with the slow waves of the arterial pressure, and high frequency (HF) - close to $0.25 \mathrm{~Hz}$ - related with respiration (Lombardi et al., 1996). For series A both components are distinct. However, addition of EMG noise to the analyzed ECG signal resulted in numerous measurement errors which spoiled not only the time properties of the determined RTp series, but also deformed their power spectra. Only after projective filtering (D) the two highest spectral peaks are close to the original ones (A). Thus, nonlinear projective filtering allowed most reliable analysis of the repolarization duration variability also in the frequency domain.

\subsection{NPF for fetal ECG extraction}

An important area of projective filtering applications is related with processing and analysis of the maternal abdominal electric signals for extraction of the fetal component (Richter et al., 1998; Kotas, 2007b). NPF can be combined with spatial approaches to achieve FECG signal enhancement (Kotas, 2008a) or even to perform separation of the multiple FECG signals in twin pregnancies (Kotas et. al., 2008b). In this chapter, we study the influence of NPF on the results of fetal QRS complexes detection. This issue is of high significance, because the noninvasive fetal electrocardiography offers higher accuracy of fetal heart rate monitoring than the routine instrumentation based on the Doppler ultrasound technique (Jeżewski, Wrobel \& Horoba, 2006). The applied detection method consists of linear bandpass filtering for noise attenuation, differentiation for QRS slopes estimation, squaring and moving window integration and, finally, comparison with the threshold for QRS peaks localization (Kotas et al, 2008a).

As it was mentioned in \$5.1, separation of the maternal and the fetal ECG in a singlechannel signal can be accomplished in two stages. First, projective filtering is applied to suppress the fetal component and to estimate the maternal ECG. Then, the estimated MECG is subtracted from the original composite signal and this way suppressed. As a result, the 
fetal component is extracted. This procedure was illustrated in Fig.10. The uppermost, on both sides of the figure, is the composite maternal abdominal signal obtained after high-pass filtering. Since fetal QRS detection was of interest, and the requirements limiting distortions of the desired component did not have to be satisfied, a relatively high cut-off frequency of 5 $\mathrm{Hz}$ was applied. The results of MECG enhancement by application of PFTAB (the most appropriate for FECG extraction, as we inferred in \$5.1) are presented below on the left; the FECG extracted - on the right.

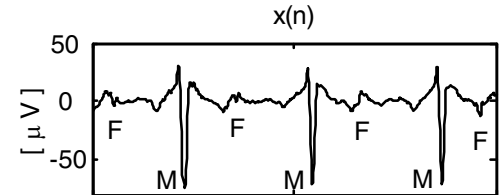

A) $x^{\prime}(n), \quad$ PFTAB: $m=50, q=0$

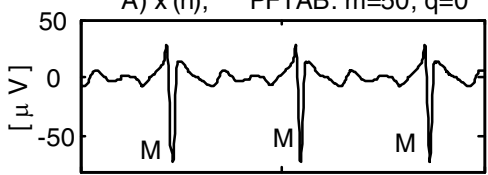

B) $x^{\prime}(n), \quad$ PFTAB: $m=50, q=1$

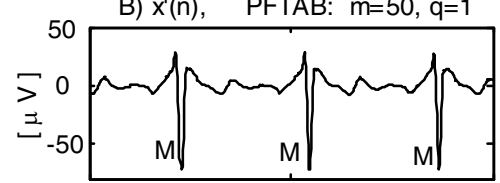

C) $x^{\prime}(n), \quad$ PFTAB: $m=50, q=2$

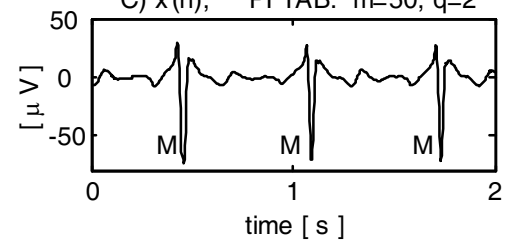

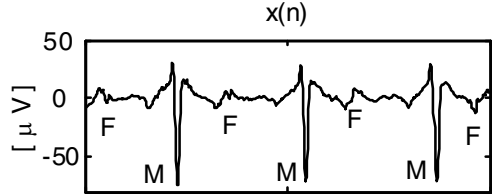

A) $x(n)-x^{\prime}(n)$

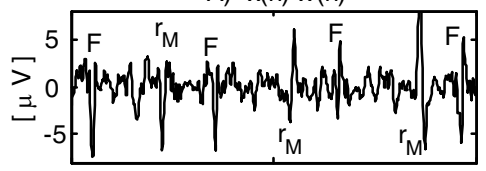

B) $x(n)-x^{\prime}(n)$

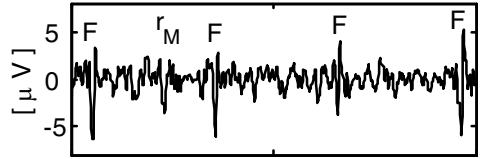

C) $x(n)-x^{\prime}(n)$

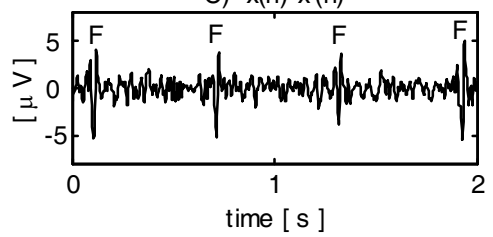

Fig. 10. The maternal abdominal signal (the uppermost on both sides of the figure) and the results of MECG enhancement (on the left) and FECG extraction (on the right): F marks the fetal QRS complexes, M-the maternal ones and $\mathrm{r}_{\mathrm{M}}$-residua of the latter.

The figure illustrates the influence of the dimension $q$ of local signal subspaces on MECG enhancement and FECG extraction. For $q=0$, the operation of PFTAB is similar to timeaveraging, and the filter is not able to preserve the morphological changes of the respective maternal QRS complexes. As a result, the operation of subtraction produced the FECG signal corrupted with the residua of these complexes. For $q=1$, the residua were significantly smaller, and for $q=2$ they almost vanished. Unfortunately, for higher values of $q$ projective filtering preserves some residua of fetal QRS complexes in the estimated MECG signal, and the subtraction leads to inconvenient suppression of these complexes. Different dimensions of local signal subspaces can be applied for different positions within a beat to improve the results of FECG extraction (Kotas, 2007b); however, this study is limited to the basic approach, and the constant dimensions are used. 
For evaluation of PFTAB influence on the results of fetal QRS detection, ten four-channel signals (five minutes long) were used. For each signal, the fetal QRS complexes locations were marked manually and stored. In the experiments, PFTAB was applied with $c_{R}=0.25$, $\tau_{m}=200 \mathrm{~ms}$ and $q$ varied from 1 to 3 to FECG extraction in the respective channels of the test signals. The test detections were performed in each channel separately.

Comparing the locations of the detected complexes with the previously stored reference ones, we can establish the number of missed complexes $\left(N_{M}\right)$ and the number of false alarms $\left(N_{F A}\right)$. Results of the detection tests are evaluated with the following performance index (Hamilton \& Tompkins, 1986)

$$
P I=\frac{N-N_{F A}-N_{M}}{N} \cdot 100[\%]
$$

The obtained indices are presented in Table. 1.

\begin{tabular}{|c|c|c|c|c|c|}
\hline PI [ \% ] & c1 & c2 & c3 & c4 & c1-c4 \\
\hline PFTAB: q=1 & 93.3 & 96.8 & 94.1 & 79.2 & 90.9 \\
\hline PFTAB: q=2 & 94.2 & 96.5 & 95.9 & 83.4 & 92.5 \\
\hline PFTAB: q=3 & 93.0 & 96.1 & 95.4 & 80.7 & 91.3 \\
\hline TS & 93.3 & 95.8 & 93.0 & 82.4 & 91.1 \\
\hline AICF & 92.1 & 96.3 & 91.5 & 75.9 & 89.0 \\
\hline
\end{tabular}

Table 1. Performance indices obtained for different methods of MECG suppression in the respective channels of the test signals and for all channels together.

For reference the detection tests were performed in the FECG signals extracted by application of the method of template subtraction (TS) and the method based on the adaptive impulse correlated filter (AICF). TS performs maternal QRS complexes detection, synchronization, time-averaging, scaling and subtraction. Moreover, it is modified by the additional operations (e.g. subtraction of the template derivative) decreasing the MECG residua and preventing the FECG suppression in cases of the maternal and the fetal complexes coincidence (Matonia et al., 2006). The second reference method employs AICF (Laguna et al. 1992) instead of PFTAB to MECG signal enhancement and then performs the operation of subtraction for FECG extraction. Before application of this method, the test signals, originally stored with the sampling frequency of $500 \mathrm{~Hz}$, were resampled with the frequency of $1000 \mathrm{~Hz}$ to decrease the synchronization errors. After MECG suppression, the extracted FECGs were again resampled with the original frequency.

During recording of the test signals, the repeatable arrangement of electrodes on a surface of a maternal abdomen was applied (as described in (Kotas et al., 2008a)). Analyzing Table 1, we can infer that among the recorded channels, the second one most often contained the fetal ECG of the best quality. Since for high amplitude fetal QRS complexes the very precise suppression of the maternal ones is not crucial, in this channel the similar performance indices were obtained for all compared methods of MECG suppression. The best results were achieved by PFTAB applied with $q=1$. In the fourth channel, however, the amplitude of the fetal complexes was most often rather low, and the precision of MECG suppression was of high significance. For this channel, the differences among the compared methods were 
the greatest, and PFTAB applied with $q=2$ was most effective. It was also most effective in the first and the third channel. We can conclude that among the compared methods of MECG suppression, the one based on projective filtering of time-aligned beats allows most effective detection of fetal QRS complexes in the signals of poor quality. This result is of high significance, because very often the diagnostic analysis of the signals from the maternal abdominal wall is hindered by difficulty in performing successful detection of the fetal QRS complexes.

\section{Conclusion}

Research in the field of nonlinear dynamics resulted in development of the method of nonlinear state-space projections, which can help to solve the very crucial problems related with ECG signals processing. The method forms the multidimensional state-space representation of the processed one-dimensional signals, determines the neighborhoods of all state-space points and creates the signal subspaces for the respective of them. Each statespace point undergoes projection into its individual signal subspace. Averaging the introduced corrections leads to reconstruction of the processed signal one-dimensional representation. NSSP method allows suppressing noise whose spectrum overlaps that of the desired signal. Moreover, it can be applied to the separation of signal components that are not separable in the frequency or time domain, such as the maternal and the fetal ECG contained in the maternal abdominal signals. However, a severe shortcoming of this method is its high computational cost. It results mostly from the necessity to create the signal subspaces for each state-space point individually.

This chapter describes the modifications of the NSSP method that highly decreased its costs and adjusted its operation to the particular properties of the processed ECG signals and, as a result, increased its performance in this field of application. The modifications are based on different approaches to the determination of neighborhoods. Linear time-alignment of the ECG beats allowed constructing the signal subspaces for the successive positions within a beat only. This significantly reduced the computational costs. Moreover, the developed method (PFTAB) almost completely cancelled the influence of high energy EMG noise, or the other signal components apart from the dominant one on determination of the neighborhoods. The performed experiments have proved that projective filtering of timealigned ECG beats can successfully be applied to fetal ECG extraction from the maternal abdominal signals for fetal heart rate determination.

Application of nonlinear alignment of the sequences of state-space points to neighborhoods determination made the method more flexible. Although projective filtering of time warped ECG beats is less immune than PFTAB to such signal components as the fetal ECG, it allows more precise reconstruction of the desired ECG embedded in EMG noise. Applied to signal enhancement prior to the analysis of the dynamic changes of the low level ECG waves, it allowed precise evaluation of the beat-to-beat variability of the repolarization duration both in time and frequency domain. The method can also be applied prior to the measurements of other indices quantifying the ventricular repolarization. This issue will be a topic of further study. 


\section{Acknowledgment}

The author thanks Dr Janusz Jeżewski and the staff from the Institute of Medical Technology and Equipment, ITAM, for the access to the database of the maternal abdominal ECG signals.

\section{References}

Chou, H. H., Chen, Y. J., Shiau, Y. C. \& Kuo T. S. (2006), An Effective and Efficient Compression Algorithm for ECG Signals With Irregular Periods", IEEE Trans. Biomed. Eng., Vol. 53, pp. 1198-1205, ISSN: 0018-9294.

$\mathrm{Hu}, \mathrm{X} ., \mathrm{Nenov}, \mathrm{V}$. (2006) A Single-Lead ECG Enhancement Algorithm Using a Regularized Data-Driven Filter, IEEE Trans. Biomed. Eng., Vol. 53, pp. 347-351, ISSN: 0018-9294.

Hamilton, P. S. \& Tompkins, W. J. (1986) Quantitative investigation of QRS detection rules using the MIT/BIH arrhythmia database, IEEE Trans. Biomed. Eng., Vol. 33, pp. 1157-1165, ISSN:0018-9294.

Jane, R.; Rix, H., Caminal, P. \& Laguna, P. (1991) Alignment Methods for Averaging of High Resolution Cardiac Signals: A Comparative Study of Performance, IEEE Trans. Biomed. Eng., Vol. 38, pp. 571-579, ISSN:0018-9294.

Jeżewski, J., Wrobel, J., \& Horoba, K. (2006) Comparison of Doppler Ultrasound and Direct Electrocardiography Acquisition Techniques for Quantification of Fetal Heart Variability, IEEE Trans. Biomed. Eng., Vol. 53, pp. 855-864.

Kotas, M. (2004) Projective Filtering of Time-Aligned ECG Beats, IEEE Trans. Biomed. Eng., Vol. 51, pp. 1129-1139, ISSN:0018-9294.

Kotas M. (2006) Application of projection pursuit based robust principal component analysis to ECG enhancement, Biomed. Signal Process. Control, Vol. 1, pp. 289-298.

Kotas M. (2007a) Projective filtering of time-aligned ECG beats for repolarization duration measurement, Computer Methods and Programs in Biomedicine, Vol. 85, No. 2, pp. 115123, ISSN:0169-2607.

Kotas M. (2007b) Projective Filtering of Time-Aligned Beats for Foetal ECG extraction, Bulletin of the Polish Academy of Sciences, Technical Sciences, Vol. 55, No.4,pp. 331-339.

Kotas M. (2008a) Combined Application of Independent Component Analysis and Projective Filtering to Fetal ECG Extraction, Biocybernetics and Biomedical Engineering, Vol. 28, No. 1, pp. 75-93.

Kotas M. (2008b) Projective filtering of time warped ECG beats, Computers in Biology and Medicine, Vol. 38, No. 1, pp. 127-37, ISSN:0010-4825.

Kotas M. (2008c) Robust Projective Filtering of Time Warped ECG Beats, Computer Methods and Programs in Biomedicine, Vol. 92, No. 10, pp. 161-172, ISSN:0169-2607

Kotas, M., Jeżewski, J., Kupka, T. \& Horoba K. (2008a) Detection of low amplitude fetal QRS complexes, in Proc. of 30th IEEE/EMBS Conference, pp. 4764-4767.

Kotas, M., Jeżewski, J., Matonia, A. \& Kupka T. (2008b) Separation of abdominal fetal electrocardiograms in twin pregnancy, Journal of Medical Informatics and Technologies, Vol. 12, pp. 83-90.

Laguna, P., Thakor, N.V., Caminal, P., Jane, R., Yoon, H. (1990) New Algorithm for QT Interval Analysis in 24 Hour Holter ECG: Performance and Applications, Med. Biol. Eng Comp., Vol. 28, pp. 67-73. 
Laguna, P., Jane, R., Meste, O., Poon, P. W., Caminal P., Rix, H., Thakor, N. V. (1992) Adaptive Filter for Event-Related Bioelectric Signals Using Impulse Correlated Reference Input: Comparison with Signal Averaging Techniques, IEEE Trans. Biomed. Eng., Vol.39, pp. 1032-1043, ISSN: 0018-9294

Laguna, P., Mark, R.G., Goldberg, A., \& Moody, G.B. (1997) A Database for Evaluation of Algorithms for Measurement of QT and Other Waveform Intervals in the ECG, Proceedings of Int. Conf. Computers in Cardiology, pp. 673-676, ISBN 0-7803-4445-6, Lund, Sweden, Sep. 1997.

Lim, J. S., Oppenheim, A. V. (1988) Advanced Topics in Signal Processing, Prentice Hall, ISBN: 0130131296, Englewood Cliffs, New Jersey, USA.

Lombardi, F., Sandrone, G., Porta, A., Torzillo, D., Terranova, G., Baselli, G., Cerutti, S. \& Malliani, A. (1996) Spectral analysis of short term R-Tapex interval variability during sinus rhythm and fixed atrial rate, European Heart Journal, Vol. 17, pp. 769778.

Malik, M., \& Batchvarov, V. (2000) Measurement, Interpretation and Clinical Potential of QT Dispersion, J. Am. Coll. Cardiol., Vol. 36, pp. 1749-1766.

Matonia, A., Jeżewski, J., Horoba, K., Gacek, A. (2006) The Maternal ECG Suppression Algorithm for Efficient Extraction of the Fetal ECG from Abdominal Signal, Proc. IEEE EMBS Conf., New York City, USA, pp. 3106-3109.

Merri, M., Alberti, M. \& Moss, A. J. (1993) Dynamic Analysis of Ventricular Repolarization Duration from 24-hour Holter recordings, IEEE Trans. Biomed. Eng., Vol. 40, pp. 1219-25, ISSN: 0018-9294.

Olmos, S., Garcia, J., Jane, R. \& Laguna, P. (1999) ECG signal compression plus noise filtering with truncated orthogonal expansions, Signal Processing, Vol. 79,pp. 97-115, ISSN:0165-1684.

Pahlm, O. \& Sörnmo, L. (1987) Data Processing of Exercise ECG's, IEEE Trans. Biomed. Eng., Vol. 34, pp. 158-165, ISSN: 0018-9294.

Paul, J. S., Reddy, M. R. \& Kumar V. J. (2000) A transform domain SVD filter for suppression of muscle noise artefacts in exercise ECG's, IEEE Trans. Biomed. Eng., Vol. 47, No.5, pp. 654-663, ISSN: 0018-9294.

Richter, M., Schreiber, T., \& Kaplan, D. T. (1998) Fetal ECG extraction with nonlinear state space projections, IEEE Trans. Biomed. Eng., Vol. 45, pp. 133-137, ISSN: 0018-9294.

Sakoe, H. \& Chiba, S. (1978) Dynamic programming optimization for spoken word recognition, IEEE Trans. ASSP, Vol. ASSP-26, pp.43-49.

Schreiber T. (1995) Efficient neighbor searching in nonlinear time series analysis, Int. J. Bifurcation and Chaos, Vol.5, pp. 349-358.

Schreiber, T. \& Kaplan, D. (1996) Nonlinear Noise Reduction for Electrocardiograms, Chaos, Vol. 6, pp. 87-92.

Tikkanen, P. E., Sellin, L. C., Kinnunen, H. O., \& Huikuri, H. V. (1999) Using simulated noise to define optimal QT intervals for computer analysis of ambulatory ECG, Med. Eng. Physics, Vol. 21, pp. 15-25.

Wariar, R. \& Eswaran, C. (1991) Integer coefficient bandpass filter for the simultaneous removal of baseline wander, 50 and $100 \mathrm{~Hz}$ interference from the ECG, Med. Biol. Eng. Comput. Vol. 29, pp. 333-336. 


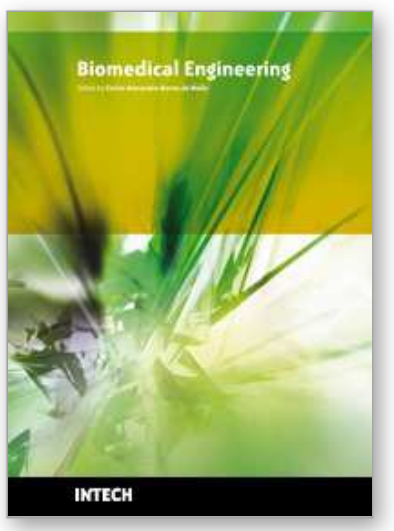

\author{
Biomedical Engineering \\ Edited by Carlos Alexandre Barros de Mello
}

ISBN 978-953-307-013-1

Hard cover, 658 pages

Publisher InTech

Published online 01, October, 2009

Published in print edition October, 2009

Biomedical Engineering can be seen as a mix of Medicine, Engineering and Science. In fact, this is a natural connection, as the most complicated engineering masterpiece is the human body. And it is exactly to help our "body machine" that Biomedical Engineering has its niche. This book brings the state-of-the-art of some of the most important current research related to Biomedical Engineering. I am very honored to be editing such a valuable book, which has contributions of a selected group of researchers describing the best of their work. Through its 36 chapters, the reader will have access to works related to ECG, image processing, sensors, artificial intelligence, and several other exciting fields.

\title{
How to reference
}

In order to correctly reference this scholarly work, feel free to copy and paste the following:

Marian Kotas (2009). Nonlinear Projective Filtering of ECG Signals, Biomedical Engineering, Carlos Alexandre Barros de Mello (Ed.), ISBN: 978-953-307-013-1, InTech, Available from:

http://www.intechopen.com/books/biomedical-engineering/nonlinear-projective-filtering-of-ecg-signals

\section{INTECH}

open science | open minds

\author{
InTech Europe \\ University Campus STeP Ri \\ Slavka Krautzeka 83/A \\ 51000 Rijeka, Croatia \\ Phone: +385 (51) 770447 \\ Fax: +385 (51) 686166 \\ www.intechopen.com
}

\author{
InTech China \\ Unit 405, Office Block, Hotel Equatorial Shanghai \\ No.65, Yan An Road (West), Shanghai, 200040, China \\ 中国上海市延安西路65号上海国际贵都大饭店办公楼 405 单元 \\ Phone: +86-21-62489820 \\ Fax: $+86-21-62489821$
}


(C) 2009 The Author(s). Licensee IntechOpen. This chapter is distributed under the terms of the Creative Commons Attribution-NonCommercial-ShareAlike-3.0 License, which permits use, distribution and reproduction for non-commercial purposes, provided the original is properly cited and derivative works building on this content are distributed under the same license. 